\title{
Síndrome de nariz vacía
}

\author{
Empty Nose Syndrome
}

Diego García P1, Natalia Jara $\mathbf{0}^{1}$, Lara Fernández $\mathbf{R}^{2}$, Alfredo Naser $\mathbf{G}^{2}$.

\section{RESUMEN}

El síndrome de nariz vacía es una enfermedad de reciente descripción, que no tiene una definición clínica clara. Se puede originar como complicación de la cirugía de resección de cornetes, en especial del inferior. La fisiopatología no está del todo definida, pero es probable que posterior a la resección de cornetes se altere la permeabilidad nasal, interfiriendo con los mecanismos neurosensitivos y con las funciones de los cornetes. Su síntoma principal, es la obstrucción nasal paradójica. El diagnóstico es clínico, basado en los síntomas con los hallazgos de una cavidad nasal amplia posterior a la cirugía nasal. El tratamiento es difícil por lo subjetivo de los síntomas, prefiriendo en un comienzo el tratamiento médico y reservando la cirugía para casos más severos. Lo más importante es la prevención, realizando cirugías lo más conservadora posibles de la anatomía de la cavidad nasal.

Palabras clave: Síndrome de nariz vacía, obstrucción nasal paradójica, turbinectomía.

\begin{abstract}
The Empty Nose Syndrome is a recently described disease, which has no clear clinical definition. It is a rare complication of turbinate surgery especially in the inferior turbinectomy. The pathophysiology is unclear, but after turbinectomy the nasal patency could be affected and this can interfere with neurosensory mechanisms and functions of the turbinates. The most common symptom is the paradoxical nasal obstruction. The diagnosis is clinical, based on symptoms with the discovery of a wide nasal cavity after nasal surgery. Treatment is difficult because of the subjective symptoms, initially preferring medical treatment and surgery is reserved for more severe cases. Most important is the prevention, being as conservative as possible with the nasal cavity.
\end{abstract}

Key Words: Empty Nose Syndrome, Paradoxical nasal obstruction, Turbinectomy.

1 Médico Cirujano. Capacitación ORL, Hospital Clínico Universidad de Chile.

2 Médico Servicio de Otorrinolaringología, Hospital Clínico Universidad de Chile.

Recibido el 26 de agosto, 2014. Aceptado el 8 de octubre, 2014. 


\section{DEFINICIÓN}

El síndrome de nariz vacía (SNV) es una entidad clínica controversial que no posee una fisiopatología del todo clara ${ }^{1}$. Se considera como una rinitis atrófica secundaria iatrogénica. En 2001, se publica el término refiriéndose a aquellos pacientes que sufren de síntomas similares a los de rinitis atrófica (RA) asociado con cavidades nasales sin cornetes identificables secundario a una intervención quirúrgica². Se podría definir como una complicación de la cirugía endonasal, particularmente de la resección parcial o total de cornetes, en especial el inferior. Esta puede aparecer meses 0 años después de la resección de cornetes. El síntoma más común es la obstrucción nasal paradójica, denominada como tal por la presencia de obstrucción en pacientes que poseen cavidades nasales objetivamente permeables ${ }^{1,3-5}$.

Houser clasifica el SNV en cuatro tipos según la forma de alteración de los cornetes. El primer tipo, se produce secundariamente a la resección total o parcial del cornete inferior. El segundo tipo, se produce secundario a la resección total 0 parcial del cornete medio. El tercer tipo, cuando tanto el cornete inferior como el medio han sufrido al menos una resección parcial. Finalmente, existe un cuarto tipo que se ve en pacientes con antecedentes de algún tipo de procedimiento en los cornetes, que tienen aparentemente cornetes de tamaño adecuado pero consultan por síntomas de SNV.

Se estima que aproximadamente hasta el 20\% de los pacientes sometidos a una resección de cornete inferior desarrollará el SNV con todos sus síntomas $^{3}$. Existen algunos trabajos israelitas que evaluaron pacientes posresección de cornetes, siguiéndolos por 6 y hasta 10 a 15 años sin mostrar síntomas de SNV, por lo que existe incertidumbre respecto a por qué algunos pacientes lo desarrollan y otros $\mathrm{no}^{7,8}$. En Chile no hay estudios epidemiológicos respecto a esta complicación quirúrgica.

\section{FISIOPATOLOGÍA}

La fisiopatología de SNV está poco dilucidada, pero existen varias hipótesis complementarias descritas en la literatura. Posterior a la cirugía endonasal, se produciría una reducción del área de la mucosa expuesta al flujo de aire, aunque la cantidad de mucosa resecada no estaría en directa relación con la aparición de SNV ${ }^{9}$. Junto con eso disminuiría el número de mecanorreceptores, receptores sensitivos, táctiles y de temperatura, resultando en la pérdida de las funciones nasales fisiológicas de humidificación, calentamiento y limpieza del aire inhalado ${ }^{4}$.

La cabeza del cornete inferior, actúa direccionando mayoritariamente el flujo aéreo al meato medio ${ }^{10}$, lo que es de gran importancia para calefaccionar y humidificar el aire inspirad $0^{5,10}$. Naftali comparó la capacidad de acondicionamiento nasal en un modelo tridimensional con y sin cornetes, removió los cornetes medio e inferior y midió la variación en humedad y temperatura del aire inspirado, encontrando una reducción en el $23 \%$ de la capacidad de humidificación y calefacción al comparar con el modelo nasal anatómicamente completo ${ }^{11}$. Esta menor humidificación del aire inspirado explicaría la sensación de sequedad que presentan los pacientes con SNV y favorecería la formación de costras, colaborando en disminuir la sensación del flujo inspiratorio y en la aparición de sequedad tanto nasal como faríngea ${ }^{3,5,9,11}$

La obstrucción nasal paradójica tendría múltiples orígenes. Esta sensación se debería a cambios fisiopatológicos que ocurren simultáneamente. Cambios como la alteración de la resistencia nasal al flujo aéreo, la generación de flujos aéreos patológicos, la pérdida de mucosa funcional, el ensanchamiento de la cavidad nasal y la disminución del contacto entre el aire y la mucosa ${ }^{1,3,5}$. El flujo de aire inspirado al ingresar a la nariz y no encontrarse con la principal resistencia (la cabeza del cornete inferior), se direcciona principalmente por el piso de la cavidad nasal. Esto evita que el aire inspirado en su mayoría entre en contacto con la mucosa, disminuyendo la velocidad de flujo, perdiéndose la interfase aire-mucosa y disminuyendo la sensación de flujo aéreo $0^{1,5,10}$.

Estos cambios a nivel de la nariz están en directa relación con la función pulmonar. La porción nasal de la respiración es responsable del 50\%$80 \%$ de la resistencia de la vía aérea. Se afecta el reflejo naso-pulmonar que determina la apertura de bronquiolos periféricos para permitir un mejor 
intercambio gaseoso, influyendo en la sensación de obstrucción $\mathrm{n}^{5,12}$.

Algunos autores han puesto en duda que exista una patología orgánica, por el hecho de que no es una complicación que aparece en todos los pacientes posteriormente a la cirugía de cornetes, sumado a series de pacientes que se siguieron por años sin la aparición del SNV, al difícil manejo y la presencia importante de síntomas psiquiátricos en estos pacientes ${ }^{1,4,9}$.

En otras cirugías de la especialidad se han objetivado síntomas similares, lo que permitiría pensar que cirugías endonasales amplias sin resección de cornetes también pudieran producir esta patología, pero hasta el momento por nomenclatura el SNV solo se atribuye a rinitis atróficas secundarias iatrogénicas por alteración conchal.

\section{DIAGNÓSTICO}

Es difícil el diagnóstico del SNV, porque no existen exámenes que objetiven la patología ${ }^{12}$, por la falta de consenso en la definición clínica, la variedad de síntomas y el factor psicosocial asociado. El diagnóstico se realiza en base al reporte subjetivo de síntomas y al examen clínico durante la consulta 9 .

La queja principal es la obstrucción nasal paradójica ${ }^{3,4,12}$. Muchas veces asociado con sensación de ahogo y dificultad para sentir el flujo del aire a través de la nariz. Otro síntoma asociado es la disminución del tiempo de inspiración (respiraciones cortas) ${ }^{3}$. Además pueden tener dolor relacionado o no con el frío, sensación de nariz vacía o sequedad rinofaríngea. Los síntomas varían en intensidad y pueden restringir las actividades cotidianas alterando la calidad de vida, lo cual puede objetivarse a través del cuestionario estandarizado SNOT-253,9.

Otros síntomas se asocian a esta condición: sensación de flujo excesivo, pérdida de la sensación de flujo aéreo nasal, hiposmia, costras, disnea, cefalea, insomnio, disminución de la capacidad de mantener la concentración, fatiga, frustración, irritabilidad, ansiedad y ánimo bajo $0^{3,9,12,13}$.

En el examen físico, se puede apreciar característicamente una cavidad nasal amplia producto de cirugía(s) previa(s), con ausencia del tejido de los cornetes. La mucosa se puede encontrar pálida, seca, eventualmente con costras. La sequedad nasal, sería un síntoma fácil de objetivar en estos pacientes ${ }^{9}$.

En algunos pacientes se puede colocar un implante para reducir el área nasal. Para corroborar diagnóstico e intentar predecir un buen resultado con dicha intervención, existe una prueba preterapéutica llamada el test del algodón. Consiste en la instalación de una pieza de algodón embebido en suero fisiológico en la cavidad nasal, en el lugar donde se instalaría el implante y luego de 20-30 minutos evaluar si existe 0 no alivio de los síntomas. Los pacientes con SNV presentan mejoría de sus síntomas ${ }^{3,12}$.

Pese que el diagnóstico es clínico, el estudio imagenológico con tomografía de cavidades paranasales tendría utilidad para la evaluación prequirúrgica. Los pacientes con SNV característicamente presentan una cavidad amplia, con pérdida de tejido de algunos cornetes (Figura 1). Además podrían presentar velamiento de senos paranasales y engrosamiento de la mucosa na$\mathrm{sal}^{13}$.

La rinomanometría tendría un rol para objetivar la ausencia de obstrucción nasal ${ }^{9}$.

\section{TRATAMIENTO}

Es una patología crónica de difícil y limitado manejo. Al ser una enfermedad secundaria a un procedimiento quirúrgico irreversible, lo principal es la prevención y se recomienda ser lo más conservador posible en las cirugías de cornetes para evitar su aparición $n^{5,9,13}$, enfatizando que esta cirugía tiene como objetivo preservar la función de la mucosa mientras se mantiene una adecuada resistencia al flujo aére ${ }^{14}$. Se deben preferir técnicas más conservadoras como la electrocauterización, radiofrecuencia, resección parcial de cornetes (menor al 50\%), resección de tejido submucoso para conservar la superficie de la mucosa y evitar la resección del cornete medio e inferior de un mismo lado $0^{9,14}$.

El manejo médico debe ser el primer enfrentamiento del SNV. Este busca mantener la mucosa nasal lo más funcional posible asegurando la humectación, eliminar factores gatillantes de sín- 


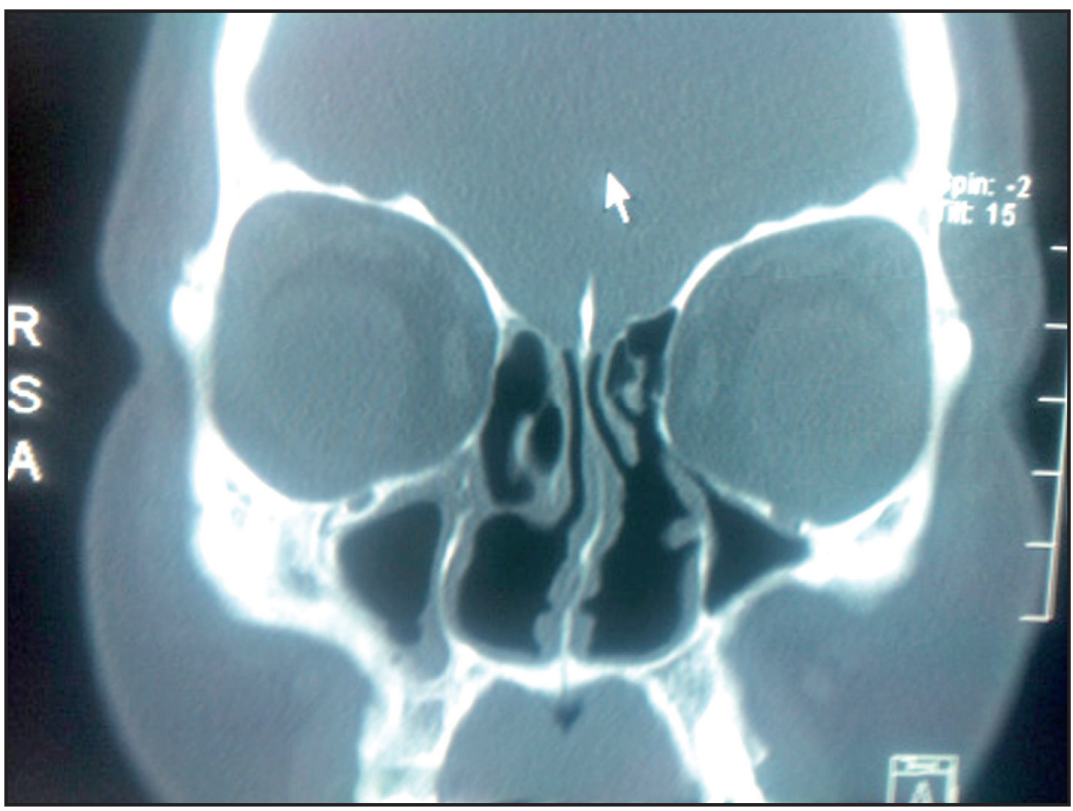

Figura 1. TC de CPN en paciente con síndrome de nariz vacía que objetiva turbinectomía bilateral.

tomas, evitar la aparición de costras y remoción de éstas si están presentes, mantener la limpieza de la mucosa y tratar las eventuales infecciones ${ }^{14}$.

Según la revisión de Hildenbrand ${ }^{14}$, el uso de soluciones salinas (isotónicas o hipertónicas), ya sea como irrigación o aerosol, resultan indispensables en el manejo del SNV, con el objetivo de humectar la mucosa; siendo especialmente útiles en el manejo de la sensación de sequedad nasal y en la formación recurrente de costras. Los ungüentos intranasales, reducirían la pérdida de agua nasal al igual que la aplicación de glicerol al 10\%. El uso tópico de dexpantenol (análogo del ácido pantoténico), dismuiría la pérdida de agua y estimularía la reepitelización por activación de fibroblastos. La inhalación de aceites nasales (sésamo, soya, maní, lavanda, eucalipto, tomillo y mentol), en concentraciones entre 0,2\%-2\%, mejorarían la motilidad ciliar, tendrían un mayor efecto humectante que las soluciones salinas, mejorarían la sequedad nasal y la obstrucción nasal paradójica, sin embargo podrían producir mal olor, prurito y goteo nasal ${ }^{4,14}$.

En el caso de aparición de costras y sobreinfección bacteriana, el uso de soluciones salinas debe ir acompañado de descostraje y de antibióticos recomendándose el uso de ciprofloxacino 500-750 mg cada 12 horas por 8 semanas $5^{5,9,14}$.

El tratamiento quirúrgico estaría indicado en aquellos pacientes con gran alteración en la calidad de vida y en quienes el manejo médico no tiene beneficios. No se conocen cifras de éxito ni fracaso posterior a manejo médico dado que no ha sido estandarizado, por lo que la indicación quirúrgica en muchos pacientes será relativa. Dicha indicación debe ser cuidadosa y estaría recomendado apoyo neuropsicológico del paciente. La cirugía en el SNV tiene como objetivo reducir el espacio de la cavidad nasal para aumentar la resistencia al flujo aéreo, incrementar la humidificación del aire y desviar el aire hacia mucosa sana que no ha sido expuesta a cirugía. La cirugía consiste en implantar un neocornete de distintos materiales en la cavidad nasal, ya sea en el tabique, piso o pared lateral de ésta6,9. Implantes autólogos de cartílago costal o septal han mostrado resultados satisfactorios, Jang ${ }^{13}$ reportó una serie de 12 pacientes con un seguimiento promedio de 12 meses, en los que se evaluó severidad del SNV mediante una escala visual análoga pre y poscirugía a los seis y 12 meses, nueve de ellos 
mostraron mejoría de los síntomas al finalizar el seguimiento.

También hay implantes de materiales sintéticos (hydroxiapatita, goretex, teflón) logrando un aumento de volumen de los cornetes. Houser describió una técnica de implante submucoso con dermis acelular, material que sería más predecible, y moldeable que sintéticos. La ubicación del implante dependería del tipo de SNV del paciente y del resultado el test de algodón. Este último permitiría definir el tamaño y ubicación del neocornete ${ }^{12}$. Houser, realizó seguimiento por un periodo variable de seis meses a cuatro años a solo ocho individuos con SNV en quienes realizó implante submucoso de dermis acelular, mediante SNOT-20 y texto libre pre y poscirugía. En ellos encontró una reducción significativa del puntaje en la encuesta (56 versus 37 puntos) y los pacientes refirieron mejoría de los síntomas en forma variable entre $0 \%$ hasta el $80 \%$ en el mejor de los casos ${ }^{12}$.

Si bien las series de Jang $^{13}$ y Houser ${ }^{12}$ son pequeñas y dejan algunas dudas sobre la metodología que se usó, dan indicios de posibles tratamientos quirúrgicos.

Los pacientes con un test de algodón negativo, son malos candidatos para la cirugía ${ }^{3}$. Aquellos pacientes con resección del cornete inferior, se les puede implantar dermis acelular en el tabique en el piso 0 en la pared lateral, si se realiza esta última técnica se debe tener precaución de no obstruir el conducto nasolacrimal. Si es por ausencia del cornete medio, se debe evitar realizar el implante en la pared lateral por la eventual obstrucción del drenaje del meato medio. Si corresponde al tercer tipo de SNV, se recomienda un implante único en el tabique, que cubra el área tanto del cornete medio y del inferior. Para el cuarto tipo de SNV, Houser recomienda implantes de dermis acelular en el cornete inferior para expandir el espacio submucosa y bastaría con expandir solo la porción anterior ${ }^{3,12}$.

Se ha propuesto el uso de ácido hialurónico que por su simplicidad y seguridad podría considerarse como herramienta para casos leves, teniendo presente que no genera cambios permanentes en la fosa ${ }^{9,15}$.

Excepcionalmente en el SNV por resección de cornete medio, en pacientes en quienes los síntomas son caracterizados por dolor más que obstrucción, Houser plantea la teoría que se bene- ficiarían de un bloqueo del ganglio pterigopalatino en lugar del implante'.

\section{CONCLUSIONES}

El SNV es una complicación quirúrgica, de diagnóstico clínico, secundaria a la resección parcial o total de los cornetes medio e inferior, principalmente de este último. Se caracteriza por la sensación subjetiva de obstrucción nasal en una cavidad nasal objetivamente amplia. Su incidencia está poco definida, y tanto en el medio local como en el internacional faltan estudios que la busquen dirigídamente y determinen su epidemiología. Su fisiopatología no está del todo definida. Se cree que confluyen múltiples alteraciones fisiopatológicas desde lo macro como las modificaciones anatómicas que modifican la física del aire inspirado hasta alteraciones a nivel de propioceptores. Esta patología es un llamado de atención para quienes realizan cirugía de cornetes, para ser lo más conservadores posible con el fin de prevenirla ya que genera alteraciones importantes en la calidad de vida de quienes la padecen y tiene un manejo dificultoso y costoso.

\section{BIBLIOGRAFÍA}

1. Payne SC. Empty Nose Syndrome: What are We Really Talking About? Otolaryngol Clin North Am 2009; 42(2): 331-7.

2. EJ M, EB K. Atrophic rhinitis: a review of 242 cases. Am J Rhinol 2000; 15(6): 355-61.

3. Chhabra N, Houser SM. The Diagnosis and Management of Empty Nose Syndrome. Otolaryngol Clin North Am 2009; 42(2): 311-30.

4. Freund W, Wunderlich AP, Stöcker T, Schmitz BL, Scheithauer M0. Empty nose syndrome: Limbic system activation observed by functional magnetic resonance imaging. The Laryngoscope 2011; 121: 2019-25.

5. Scheithauer MO. Surgery of the turbinates and «empty nose» syndrome. GMS Curr Top Otorhinolaryngol Head Neck Surg 2010; 9: 1-28.

6. HOUSER S. Empty nose syndrome associated with middle turbinate resection. Otolaryngol Head Neck Surg 2006; 135(6): 972-3. 
7. Ophir D, Schindel D, Halperin D, Marshak G. Longterm follow-up of the effectiveness and safety of inferior turbinectomy. Plast Reconstr Surg 1992; 90(6): 980-4.

8. Talmon Y, Samet A, Gilbey P. Total inferior turbinectomy: operative results and technique. Ann Otol Rhinol Laryngol 2000; 109(12 Pt 1): 1117-9.

9. Coste A, Dessi P, Serrano E. Empty nose syndrome. Eur Ann Otorhinolaryngol Head Neck Dis 2012; 129(2): 93-7.

10. Simmen D, Scherrer JL, Moe K, Heinz B. A dynamic and direct visualization model for the study of nasal airflow. Arch Otolaryngol Neck Surg 1999; 125(9): 1015-21.

11. Naftali S, Rosenfeld M, Wolf M, Elad D. Pathophysiology of nasal airconditioning. Engineering in Medicine and Biology $24^{\text {th }}$ Annual
Conference and the Annual Fall Meeting of the Biomedical Engineering Society EMBS/BMES Conference Proceedings of the Second Joint 2002; 1523-4.

12. Houser SM. Surgical treatment for empty nose syndrome. Arch Otolaryngol Neck Surg 2007; 133(9): 858-63.

13. Jang YJ, Kim JH, Song HY. Empty nose syndrome: Radiologic Findings and Treatment Outcomes of Endonasal Microplasty Using Cartilage Implants. The Laryngoscope 2011; 121(6): 1308-12.

14. Hildenbrand T, Weber RK, Brehmer D. Rhinitis sicca, dry nose and atrophic rhinitis: a review of the literature. Eur Arch Otorhinolaryngol 2010; 268(1): 17-26.

15. Modrzynskı M. Hyaluronic acid gel in the treatment of empty nose syndrome. Am J Rhinol Allergy 2011; 25(2): 103-6. 